\title{
CORRIGENDUM
}

\section{Experimental EPR-steering using Bell-local states}

\section{J. Saunders, S. J. Jones, H. M. Wiseman and G. J. Pryde}

Nature Physics 6, 845-849 (2010); published online: 19 September 2010; corrected after print: 2 November 2011.

The authors wish to point out that there were systematic errors in some of the demonstrations of Alice's optimal attempt to cheat (orange lines in Fig. 5, for $n=3,4$ and 6), due to misalignment of the waveplates. The data have been retaken with the corrected settings and are included in the following corrected figure (the plot for $n=2$ is unchanged). The arguments of the paper are unaffected by this correction. These changes have been made in the PDF and HTML versions of this Letter.
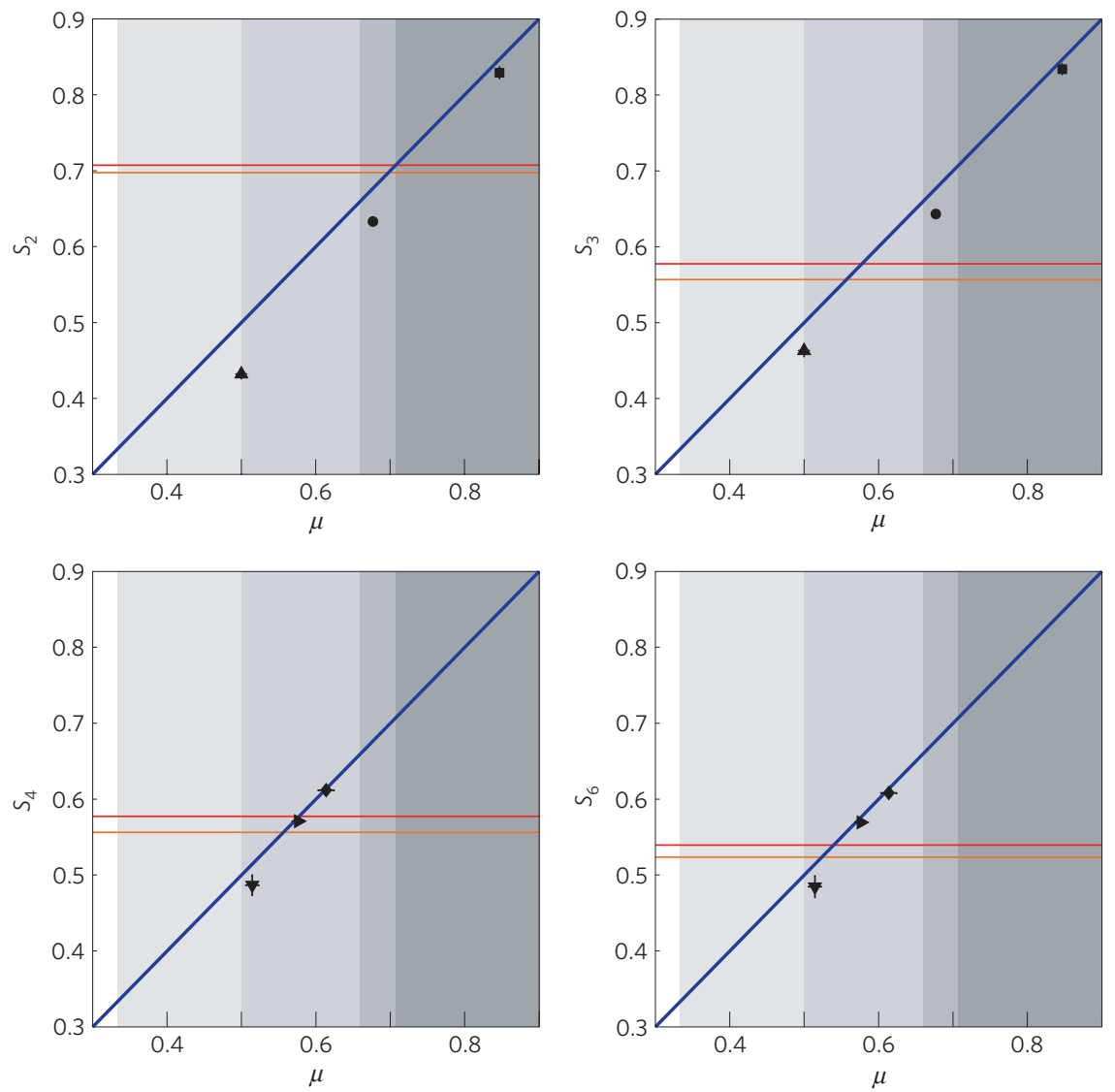